\title{
Muscle NT-3 levels increased by exercise training contribute to the improvement in caudal nerve conduction velocity in diabetic rats
}

\author{
HONGWEI LI ${ }^{1,2^{*}}$, ZHENHAI SHEN ${ }^{1 *}$, YUN LU $^{1}$, FENG LIN ${ }^{2}$, YAWEN WU ${ }^{2}$ and ZHONGLI JIANG ${ }^{2}$ \\ ${ }^{1}$ Department of Rehabilitation Medicine, Jiangsu Lake Taihu Cadres Sanatorium \\ of Jiangsu Provincial People's Hospital Group, Wuxi, Jiangsu 214086; ${ }^{2}$ Department of Rehabilitation Medicine, \\ First Affiliated Hospital of Nanjing Medical University, Nanjing, Jiangsu 210029, P.R. China
}

Received January 12, 2012; Accepted April 10, 2012

DOI: $10.3892 / \mathrm{mmr} .2012 .897$

\begin{abstract}
The aim of the present study was to explore the role of exercise in diabetic peripheral neuropathy (DPN) and the mechanisms involved. For this purpose, 31 male SpragueDawley rats were used. The rats were assigned to 5 groups: diabetic rats subjected to exercise training (swimming) for 8 weeks (D-Ex1 group), diabetic rats subjected to exercise training for 4 weeks after 4 weeks of being sedentary (D-Ex2 group), diabetic rats which remained sedentary for 8 weeks (D-Sed group), control rats subjected to exercise training for 8 weeks (C-Ex1 group) and control rats which remained sedentary for 8 weeks (C-Sed group). Blood glucose levels and caudal nerve conduction velocity ( $\mathrm{NCV}$ ) were evaluated at 0 (baseline), 28 (4 weeks) and 56 days (8 weeks) after the induction of diabetes. The levels of neurotrophin-3 (NT-3) in skeletal muscle were measured by ELISA at the end of the experiment. Blood glucose levels in the D-Ex1 group rats decreased significantly after 8 weeks of exercise. The caudal NCV markedly decreased in all diabetic rats and significantly increased after 4 or 8 weeks of exercise training. Muscle NT-3 levels were significantly lower in the D-Sed compared to the 4 other groups. Muscle NT-3 levels positively correlated with caudal NCV. In conclusion, swimming training has a beneficial effect on DPN and muscle NT-3 levels, which could help improve caudal NCV in streptozotocin-induced diabetic rats.
\end{abstract}

\section{Introduction}

Diabetic peripheral neuropathy (DPN) is one of the most common complications of diabetes and is responsible for $50-75 \%$ of non-traumatic amputations in developed

Correspondence to: Dr Zhongli Jiang, Department of Rehabilitation Medicine, The First Affiliated Hospital of Nanjing Medical University, 300 Guangzhou Road, Nanjing, Jiangsu 210029, P.R. China E-mail: jiangzh3721@yeah.net

${ }^{*}$ Contributed equally

Key words: exercise training, diabetic peripheral neuropathy, caudal nerve conduction, velocity, neurotrophin-3 countries $(1,2)$. The streptozotocin (STZ) model is widely used to investigate experimental DPN (3). Abnormal nerve conduction is an early feature of diabetic nerve damage (4). Tesfaye et al showed that conduction velocity significantly increased after exercise in normal subjects (5). Selagzi et al found that exercise training significantly increased the compound muscle action potential amplitude and decreased motor latency in diabetic rats (4). However, little is known about the mechanisms involved in exersise improving nerve dysfunction in STZ-induced diabetic rats.

Neurotrophin-3 (NT-3) is a type of neurotrophic factor that comprises a heterogeneous group of molecules produced by neurons, Schwann cells and end organs in the central and peripheral nervous system (PNS). In adults, neurotrophins of the PNS are synthesized in and released from target tissues. Skeletal muscles are such target tissues and produce neurotrophins. NT-3 plays a crucial role in mediating central nervous system plasticity and regeneration in the spinal cord and muscle. Short-term STZ-induced diabetes causes a reduction in muscle NT-3 mRNA and protein expression, which leads to a decreased retrograde axonal transport of NT-3 to the neuronal cell body and consequent sub-optimal neurotrophic support (6). Previous studies have shown that the continuous production of NT-3 by the latency-associated promoter 2 (LAP2)-driven expression of the transgene from a herpes simplex virus (HSV) vector over a 6-month period protects against the progression of diabetic neuropathy in mice (7), and treatment with NT-3 improves the nerve conduction velocity (NCV) of both the large motor and sensory fibres in STZ-induced diabetic rodents (8). These results suggest that NT-3 may provide a novel treatment for preventing the progression of diabetic neuropathy.

Skeletal muscle secretes a number of neurotrophic factors, including NT-3 (9). Ying et al found that voluntary exercise increased NT-3 mRNA levels and the expression of its receptor, tyrosine kinase $\mathrm{C}$ ( Trk $\mathrm{C}$ ), in the soleus muscle of normal rats (10). However, it is not yet clear whether or not exercise training increases the secretion of NT-3 in the skeletal muscle of diabetic rats to prevent the development of DPN. Therefore, the aim of the present study was to investigate the efficacy of physical exercise in treating DPN and the mechanisms involved. We hypothesized that muscle NT-3 levels increased by exercise training may contribute to the restoration of impaired $\mathrm{NCV}$ in STZ-induced diabetic rats. 


\section{Materials and methods}

Animals. In total, 31 male Sprague-Dawley (SD) rats (age, 12 weeks old; body weight, 250-300 g) were purchased from Shanghai SLC Co. (Shanghai, China) and were acclimated for 1 week prior to the experiment. The rats were housed in plastic cages in a temperature-controlled room $\left(23 \pm 3^{\circ} \mathrm{C}\right)$ with alternating 12 -h periods of light and dark and were allowed free access to chow and water. The study was approved by the Animal Ethics Committee of Nanjing Medical University, Nanjing, China.

Methods. In total, 19 SD rats were fasted for a period of $24 \mathrm{~h}$ and then hyperglycaemia was induced by a single intraperitoneal injection of $55 \mathrm{mg} / \mathrm{kg}$ of STZ dissolved in citrate buffer (0.1 M, pH 4.5). Blood glucose levels were measured $72 \mathrm{~h}$ following the induction of diabetes. The diabetic state was confirmed when the blood glucose concentration exceeded $16.7 \mathrm{mmol} / \mathrm{l}$.

Diabetes was successfully induced in the rats with STZ and they were randomly divided into a diabetic group subjected to exercise training for 8 weeks (D-Ex1 group, $n=6$ ), a diabetic group subjected to exercise training for 4 weeks after 4 weeks of being sedentary (D-Ex2 group, $\mathrm{n}=7$ ) and a diabetic group that remained sedentary for 8 weeks (D-Sed group, $n=6$ ). Twelve SD rats were injected with the same volume of citrate buffer as the diabetic rats and randomly divided into a control group subjected to exercise training for 8 weeks (C-Ex1 group, $\mathrm{n}=6$ ) and a control group that remained sedentary for 8 weeks (C-Sed group, $\mathrm{n}=6$ ).

Exercise was undertaken by swimming without a load in a barrel filled with water kept at $33-35^{\circ} \mathrm{C}$ to a depth of $40-50 \mathrm{~cm}$, which was large enough for each rat to swim freely $(11,12)$. The duration of the first swimming exercise was limited to $15 \mathrm{~min}$; this was increased by $15 \mathrm{~min}$ each day until the swimming session was $1 \mathrm{~h}$ long. The rats in the D-Ex1 and C-Ex1 groups swam $1 \mathrm{~h}$ per day, 5 days per week, for the 8 weeks of training. The rats in the D-Ex2 group began swimming from the 4th week until the end of the experiment. The rats which were not subjected to exercise training (D-Sed and C-Sed groups) were placed in shallow water kept at $33-35^{\circ} \mathrm{C}$, for $1 \mathrm{~h}$ per day, 5 days per week, for 8 weeks.

First, the blood glucose levels were measured in orbital vein blood samples from all 5 groups immediately prior to the induction of diabetes. Then, the blood glucose levels for the baseline data (day 0) were measured from orbital vein blood samples $72 \mathrm{~h}$ after the induction of STZ. Following this, the blood glucose levels were measured from orbital vein blood samples at 4 weeks (on the 28th day) and 8 weeks (the 56th day) into the experiment. A glucometer (SureStep Plus, LifeScan, Milpitas, CA, USA) was used to analyse the blood glucose levels.

Prior to electrophysiological recording, the rats were anaesthetized with pentobarbitone $(1.5 \%, 40 \mathrm{mg} / \mathrm{kg}$ ) administered intramuscularly (13). Caudal NCV across the nerve segment was assessed using a Keypoint electromyography (EMG)/evoked potentials (EP) system (Dantac Company, Copenhagen, Denmark). The caudal NCV was recorded at baseline and at 4 and 8 weeks in all 5 groups. The caudal $\mathrm{NCV}$ was measured at a fixed distance with the stimulating cathode positioned $120 \mathrm{~mm}$ distally to the tail hairline and the active electrode located $100 \mathrm{~mm}$ proximal to the cathode. Neural responses were induced by stimulation at a frequency of $5 \mathrm{~Hz}$. The body temperature of the rats was maintained at $37^{\circ} \mathrm{C}$ using a heating pad and continuously monitored using a rectal probe digital thermometer (13).

The rats in the D-Ex1, D-Ex2 and C-Ex1 groups were sacrificed $24 \mathrm{~h}$ after the end of the last exercise training session. The rats in the D-Sed and C-Sed groups were sacrificed after 8 weeks. After anaesthetizing the rats via an intraperitoneal injection of pentobarbitone, the quadriceps femoris from each rat was excised using 'quick-freeze' tongs, immersed in liquid nitrogen and stored at $-80^{\circ} \mathrm{C}$ until further processing (14).

Skeletal muscle samples were analysed using an NT-3 ELISA kit according to the manufacturer's instructions (No. E0106r; USCN Life Science and Technology Company, Missouri City, TX, USA) (10).

Statistical analysis. The data are presented as the means \pm SD. Statistical evaluations were performed using SPSS software (version 13.0, SPSS, Chicago, IL, USA). Data obtained during the blood glucose and caudal NCV measurements were analysed using repeated measures ANOVA. Tukey's multiple range test was used for a post-hoc analysis to detect any differences. Data from the D-Sed group were compared with those from the other groups using Dunnett's two-sided t-test. A p-value $<0.05$ was considered to indicate a statistically significant difference. Pearson's correlation co-efficient test was used to detect any correlations between the caudal $\mathrm{NCV}$ and muscle NT-3 data.

\section{Results}

All the diabetic rats and the diabetic rats subjected to exercise training presented with persistent polydipsia and polyuria. One rat in the D-Sed group succumbed after 5 weeks due to a progressive decline in body weight. Another rat in the D-Ex group drowned during the experiment.

Effects of diabetes and swimming training on body weight. No significant differences were observed in body weight between the groups at baseline. By the 4th week, the body weights of the control rats were significantly higher than those in the diabetic rats and the body weights of the C-Sed, C-Ex1 and D-Ex1 group rats were significantly higher than those at baseline. In the 8th week, the body weights of the control rats (C-Sed and C-Ex groups) continued to increase significantly and were significantly greater than those of the diabetic rats. The body weights of the D-Ex1 and D-Ex2 group rats were significantly greater by the 8 th week than at baseline (Table I).

Effects of diabetes and swimming training on blood glucose levels. The blood glucose levels at baseline were $5.45 \pm 0.29 \mathrm{mmol} / 1$ in all rats. The blood glucose levels at baseline and in the 4th and 8th weeks of the experiment were significantly higher in the diabetic rats compared to the control rats. Blood glucose levels in the 8th week in the D-Ex1 group rats were significantly lower than those in the D-Ex2 and D-Sed group rats, and were significantly higher than those in the 2 control groups (Table II). 
Table I. Body weight in all 5 groups.

\begin{tabular}{|c|c|c|c|}
\hline \multirow[b]{2}{*}{ Groups } & \multicolumn{3}{|c|}{ Body weight (kg) } \\
\hline & Baseline & 4th Week & 8th Week \\
\hline$D-\operatorname{Ex} 1(n=6)$ & $295.55 \pm 10.21$ & $356.40 \pm 42.84^{\mathrm{a}, \mathrm{b}}$ & $377.42 \pm 54.06^{\mathrm{a}, \mathrm{b}}$ \\
\hline$D-\operatorname{Ex} 2(n=7)$ & $302.06 \pm 20.86$ & $333.93 \pm 23.28^{\mathrm{a}}$ & $352.89 \pm 32.82^{\mathrm{a}, \mathrm{b}}$ \\
\hline D-Sed $(n=6)$ & $288.78 \pm 7.66$ & $301.34 \pm 33.55^{\mathrm{a}}$ & $307.80 \pm 42.38^{\mathrm{a}}$ \\
\hline C-Ex1 $(n=6)$ & $292.95 \pm 12.87$ & $413.33 \pm 10.95^{b}$ & $495.30 \pm 13.68^{b, c}$ \\
\hline C-Sed $(n=6)$ & $290.43 \pm 12.39$ & $407.97 \pm 37.80^{\mathrm{b}}$ & $489.93 \pm 35.60^{\mathrm{b}, \mathrm{c}}$ \\
\hline \multicolumn{4}{|c|}{$\begin{array}{l}\text { Values represent the means } \pm \text { SD. }{ }^{a} \mathrm{p}<0.05 \text { vs. values in the C-Ex } 1 \\
\text { and C-Sed groups by Tukey's post-hoc test. }{ }^{b} \mathrm{p}<0.05 \text { vs. values at the } \\
\text { baseline for the same group by Tukey's post-hoc test. ' } \mathrm{p}<0.05 \text { vs } \\
\text { values in the } 4 \text { th week for the same group by Tukey's post-hoc test } \\
\text { D-Ex } 1 \text {, diabetic group subjected to exercise training for } 8 \text { weeks; } \\
\text { D-Ex } 2 \text {, diabetic group subjected to exercise training for } 4 \text { weeks after } \\
4 \text { weeks of being sedentary; D-Sed, diabetic group which remained } \\
\text { sedentary for } 8 \text { weeks; C-Ex } 1 \text {, control group subjected to exercise } \\
\text { training for } 8 \text { weeks; C-Sed, control group which remained sedentary } \\
\text { for } 8 \text { weeks. }\end{array}$} \\
\hline
\end{tabular}

Table II. Blood glucose levels in all 5 groups.

\begin{tabular}{lrrr}
\hline & \multicolumn{3}{c}{ Blood glucose (mmol/l) } \\
\cline { 2 - 4 } Groups & Baseline & 4th Week & 8th Week \\
\hline D-Ex1 (n=6) & $23.40 \pm 1.61^{\mathrm{a}}$ & $20.25 \pm 5.70^{\mathrm{a}}$ & $14.98 \pm 6.30^{\mathrm{a}, \mathrm{b}}$ \\
D-Ex2 (n=7) & $21.14 \pm 2.69^{\mathrm{a}}$ & $24.72 \pm 1.95^{\mathrm{a}}$ & $20.50 \pm 4.38^{\mathrm{a}}$ \\
D-Sed (n=6) & $24.06 \pm 1.70^{\mathrm{a}}$ & $26.22 \pm 1.44^{\mathrm{a}}$ & $25.36 \pm 1.60^{\mathrm{a}}$ \\
C-Ex1 (n=6) & $5.38 \pm 0.33$ & $5.58 \pm 0.37$ & $5.77 \pm 0.29$ \\
C-Sed (n=6) & $5.52 \pm 0.29$ & $6.12 \pm 0.88$ & $5.38 \pm 0.36$ \\
\hline
\end{tabular}

Values represent the means $\pm \mathrm{SD}$. ${ }^{\mathrm{a}} \mathrm{p}<0.05$ vs. values in the $\mathrm{C}-\mathrm{Ex} 1$ and C-Sed groups by Tukey's post-hoc test. ${ }^{b} \mathrm{p}<0.05$ vs. values at the baseline for the same group by Tukey's post-hoc test. D-Ex1, diabetic group subjected to exercise training for 8 weeks; D-Ex2, diabetic group subjected to exercise training for 4 weeks after 4 weeks of being sedentary; D-Sed, diabetic group which remained sedentary for 8 weeks; C-Ex1, control group subjected to exercise training for 8 weeks; C-Sed, control group which remained sedentary for 8 weeks.

Effects of diabetes and swimming training on caudal NCV. No statistical differences in caudal NCV were found between the 5 groups at baseline. Although the caudal NCV in the 4 th week in all 3 diabetic groups was significantly lower compared to the 2 control groups, the caudal NCV had significantly increased in the D-Ex1 group compared to the D-Ex2 and D-Sed groups. The caudal NCV in the 2 diabetic groups had significantly increased with swimming training compared to the D-Sed group in the 8th week. The caudal NCV in the D-Ex1, D-Ex2, C-Ex1 and C-Sed groups had significantly increased in the 8th week compared to that at baseline, while the caudal NCV in the D-Sed group had significantly decreased in the 8th week compared to that at baseline (Table III, Fig. 1).
Table III. Caudal NCV values in all 5 groups.

\begin{tabular}{lccc}
\hline & \multicolumn{3}{c}{ Caudal NCV (m/s) } \\
\cline { 2 - 4 } Groups & Baseline & 4th Week & 8th Week \\
\hline D-Ex1 (n=6) & $37.18 \pm 5.70$ & $42.90 \pm 1.77^{\mathrm{a}, \mathrm{b}}$ & $46.80 \pm 4.40^{\mathrm{c}, \mathrm{d}}$ \\
D-Ex2 (n=7) & $34.04 \pm 1.96$ & $36.88 \pm 1.13^{\mathrm{b}}$ & $48.02 \pm 2.41^{\mathrm{c}, \mathrm{d}, \mathrm{e}}$ \\
D-Sed (n=6) & $37.92 \pm 3.25$ & $35.66 \pm 1.33^{\mathrm{b}}$ & $32.54 \pm 2.04^{\mathrm{d}}$ \\
C-Ex1 (n=6) & $33.35 \pm 2.18$ & $46.78 \pm 3.93^{\mathrm{d}}$ & $46.57 \pm 3.94^{\mathrm{c}, \mathrm{d}}$ \\
C-Sed (n=6) & $35.30 \pm 2.29$ & $46.55 \pm 3.30^{\mathrm{d}}$ & $47.73 \pm 4.54^{\mathrm{c}, \mathrm{d}}$ \\
\hline
\end{tabular}

Values represent the means \pm SD. ${ }^{\mathrm{a}} \mathrm{p}<0.05$ vs. values in the D-Ex2 and D-Sed groups by Tukey's post-hoc test. ${ }^{b} \mathrm{p}<0.05$ vs. values in the $\mathrm{C}$-Ex 1 and C-Sed groups by Tukey's post-hoc test. ${ }^{\mathrm{c}} \mathrm{p}<0.05$ vs. values in the D-Sed group by Tukey's post-hoc test. ${ }^{\mathrm{d}} \mathrm{p}<0.05$ vs. values at the baseline for the same group by Tukey's post-hoc test. ${ }^{\mathrm{e}} \mathrm{p}<0.05 \mathrm{vs}$. values in the 4th week for the same group by Tukey's post-hoc test. NCV, nerve conduction velocity. D-Ex1, diabetic group subjected to exercise training for 8 weeks; D-Ex2, diabetic group subjected to exercise training for 4 weeks after 4 weeks of being sedentary; D-Sed, diabetic group which remained sedentary for 8 weeks; C-Ex1, control group subjected to exercise training for 8 weeks; C-Sed, control group which remained sedentary for 8 weeks.

Effects of diabetes and swimming training on muscle NT-3 levels. As measured in the skeletal muscle of each sacrificed animal at the end (the 8th week) of the study, the NT-3 levels in the D-Sed group were significantly lower than those in the other 4 groups. No significant differences were found in muscle NT-3 levels between the D-Ex1, D-Ex2, C-Ex1 and C-Sed groups (Fig. 2). The muscle NT-3 levels significantly and positively correlated with the caudal NCV in the 8th week in all 5 groups $(r=0.407, n=31, p<0.026$; Fig. 3$)$.

\section{Discussion}

This study shows the protective and therapeutic effects of swimming (exercise) in rats with DPN whose diabetes had been induced with STZ. The treatment of DPN has traditionally focused on the control of hyperglycaemia (15). The impact of intensive glycaemic control on DPN has been widely evaluated (16-18). Good glycaemic control is the only way to minimize the risk of neuropathy in patients with diabetes (15). In the present study, the blood glucose levels after 8 weeks of exercise were significantly lower than the baseline levels in diabetic rats, despite the fact that these levels were still higher than those of the 2 control groups. However, exercise training for 4 weeks did not significantly reduce the blood glucose levels in diabetic rats. These results show that exercise training alone cannot reduce higher levels of blood glucose to the normal range and that the lowering of blood glucose levels also depends on the exercise training period in type 1 diabetic rats.

It is known that the body weight of diabetic rats gradually decreases as the disease progresses. Hoybergs et al reported that the body weight of low-weight rats at the beginning of the experiment gradually increased over time, while medium-weight and heavier animals showed a decrease in body weight, along with a negative correlation between body weight and body 


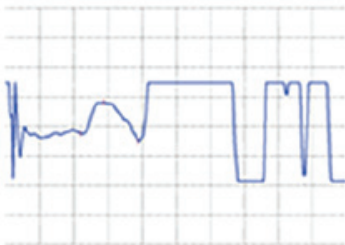

D-Ex1

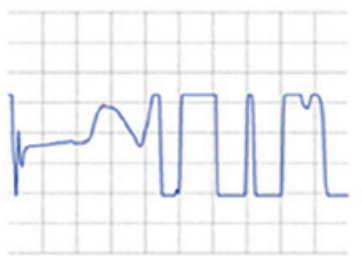

C-Ex1

Electric stimulation

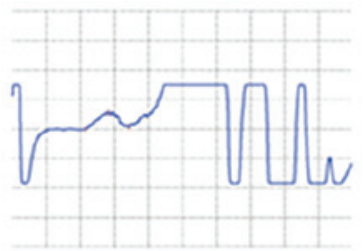

D-Ex2

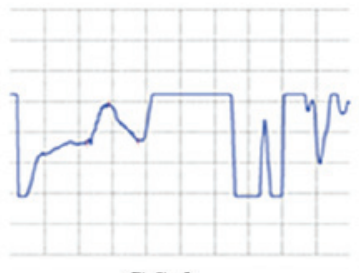

C-Sed

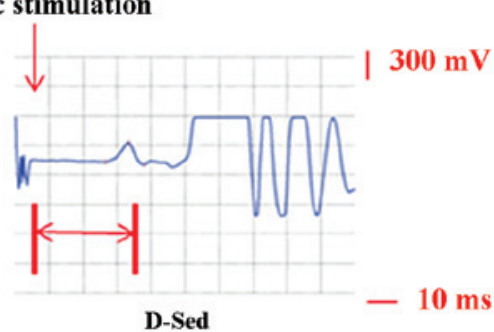

Paperfeed speed: $* * \mathrm{~min} / \mathrm{sec}$

Figure 1. Representative records of the caudal nerve conduction velocity (NCV) in the 8th week of the experiment in all 5 groups. Horizontal bar, $10 \mathrm{ms;}$ vertical bar, $300 \mathrm{mV}$. D-Ex1, diabetic group subjected to exercise training for 8 weeks; D-Ex2, diabetic group subjected to exercise training for 4 weeks after 4 weeks of remaining sedentary; D-Sed, diabetic group which remained sedentary for 8 weeks; C-Ex1, control group subjected to exercise training for 8 weeks; C-Sed, control group which remained sedentary for 8 weeks.

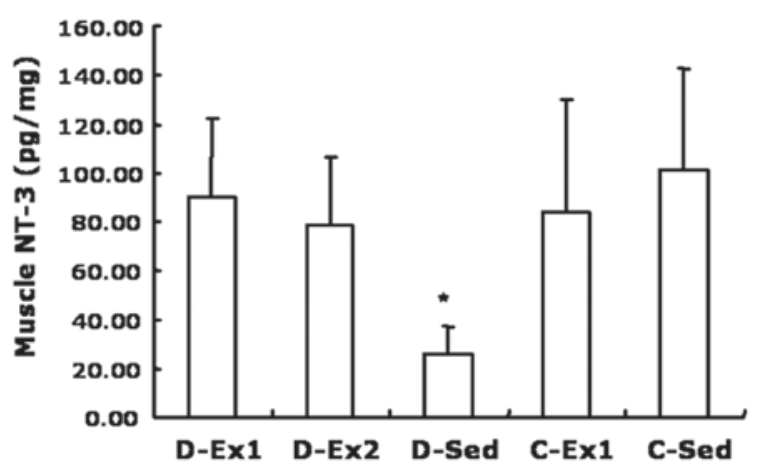

Figure 2. Muscle NT-3 levels at the end of the experiment in all 5 groups. D-Ex1, diabetic group subjected to exercise training for 8 weeks; D-Ex2, diabetic group subjected to exercise training for 4 weeks after 4 weeks of being sedentary; D-Sed, diabetic group after remaining sedentary for 8 weeks; C-Ex1, control group subjected to exercise training for 8 weeks; C-Sed, control group after remaining sedentary for 8 weeks. "Statistically significant decrease in the levels in the D-Sed compared to the D-Ex1 group ( $\mathrm{p}=0.016)$, D-Ex2 $(\mathrm{p}=0.047), \mathrm{C}-\mathrm{Ex} 1(\mathrm{p}=0.032)$ and C-Sed $(\mathrm{p}=0.005)$ by Dunnett's post-hoc test. NT-3, neurotrophin-3.

condition in the medium body weight and heavier groups (19). However, in the present study, a rapid increase in body weight was observed in the control groups (C-Ex1 and C-Sed); this was associated with overeating after exercise training. The slow increase in body weight shown by the 2 diabetic exercise groups was considered to be related to the body condition of the diabetic rats being improved by exercise, in agreement with the results reported by Selagzi et al (4).

$\mathrm{NCV}$ is used as an index of neuropathy.Zotova et al reported that the caudal NCV on the 20th day after STZ-induced hyperglycaemia had significantly decreased compared to the control rats and gradually decreased as the disease progressed (13). Tomlinson et al found that the NCV had decreased within 2 weeks of the onset of hyperglycaemia in diabetic rats (20). Our findings showed that the caudal NCV in the 4th week had

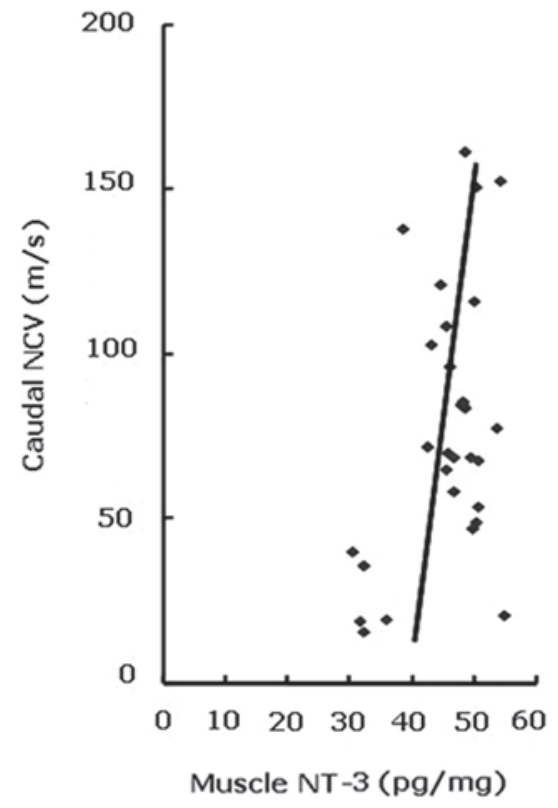

Figure 3. Pearson's correlation between caudal nerve conduction velocity (NCV) and muscle neurotrophin-3 (NT-3) levels at the end of the experiment for all 5 groups. A significant positive correlation was found between caudal NCV and muscle NT-3 (correlation co-efficient, 0.407; $\mathrm{n}=31, \mathrm{p}=0.026$ ).

significantly decreased in the diabetic rats which were not subjected to exercise (D-Sed and D-Ex2 groups) than in the other groups (D-Ex1, C-Ex1 and C-Sed), suggesting that DPN occurred at this time.

Balducci et al found that exercise training modified the natural history of DPN (21). Selagzi et al reported that swimming training for 8 weeks restored compound muscle action potential latency and prevented myelin damage (4). In the present study, it was found that starting an exercise program 8 weeks prior to the occurrence of diabetic neuropathy effec- 
tively prevented a decrease in the caudal NCV in diabetic rats (D-Ex1). Exercise training for 4 weeks also significantly accelerated caudal NCV in diabetic rats (D-Ex2), even though diabetic neuropathy had already occurred. These results indicate that exercise is effective as a protective and therapeutic method against DPN. The biological variables for NCV determination include temperature, age, height and the effects of volume conduction (22). Similar to the results from a previous study (23), in this study, it was also found that caudal NCV in the control rats significantly increased as the rats grew (23).

NT-3 plays a crucial role in the survival and functioning of sensory neurons, so much so that mice lacking the NT-3 gene show a severe loss of sensory neurons and concomitant gait abnormalities (24-26). Applying NT-3 to the spinal cord of rodents can compensate for induced damage, as shown by increased levels of axonal regeneration, reduced atrophy and increased levels of functional recovery $(6,27-30)$. In this study, muscle NT-3 levels in the diabetic groups subjected to swimming training (D-Ex1 and D-Ex2 groups) significantly increased compared to the levels in the diabetic rats which had remained sedentary (D-Sed), and they were close to the levels of the 2 control groups (C-Ex1 and C-Sed). Fernyhough et al found that the amount of NT-3 protein in hindlimb skeletal muscle was decreased by up to $70 \%$ in rats with 4-6 weeks of diabetes compared to aged-matched controls (31). These results reveal that diabetes suppresses muscle NT-3 levels and that exercise training reverses this inhibition.

Mizisin et al reported that the NCV of both large motor and sensory fibres was improved when STZ-induced diabetic rodents were treated with NT-3 (8). Chattopadhyay et al found that vector inoculation 2 weeks after the onset of diabetes was capable of preventing the development of diabetic neuropathy over the course of 6 months (7). All of these results indicate that exogenous NT-3 could have a long-term therapeutic effect. In the present study, we showed that endogenous NT-3 levels significantly and positively correlated with caudal NCV in the 8th week, suggesting that muscle NT-3 levels increased by exercise may be partly responsible for the improvement in caudal NCV in diabetic rats. Endogenous NT-3 may have certain therapeutic effects on diabetic neuropathy.

However, in this study we did not find a significant increase in muscle NT-3 levels after swimming training in the control rats. Cuppini et al showed that exercise training increases the expression of the brain-derived neurotrophic factor (BDNF) in the skeletal muscle of normal rats (9). Ying et al found that exercise increased NT-3 mRNA levels in skeletal muscle after 3 days of running-wheel training, which returned to normal 7 days later, while no significant increase was found in the amount of NT-3 protein compared to the control group (10). The muscle NT-3 levels measured by ELISA in the present study are consistent with the results presented the study by Ying et al, suggesting that physical activity has differential effects on NT-3 levels in the various muscular components of the neuromuscular system.

In conclusion, these results show that swimming training improves caudal NCV and increases muscle NT-3 levels in STZ-induced diabetic rats. The increase in muscle NT-3 levels positively correlated with caudal NCV after exercise training. This study indicates that exercise has a beneficial effect on the prevention and therapy of DPN and that the restoration of muscle NT-3 levels by exercise could be one of the main mechanisms in improving caudal NCV in diabetic rats.

\section{Acknowledgements}

This study was supported by Natural Science Foundation of Jiangsu Province (BK2011162). The authors wish to thank Dr Tokutaro Sato for thoughtful insights and suggestions. In addition, the technical assistance of Dr Lin Chen and Dr Hongxing Wang is acknowledged.

\section{References}

1. Caputo GM, Cavanagh PR, Ulbrecht JS, Gibbons GW and Karchmer AW: Assessment and management of foot disease in patients with diabetes. N Engl J Med 331: 854-860, 1994.

2. Vinik A, Ullal J, Parson HK and Casellini CM: Diabetic neuropathies: clinical manifestations and current treatment options. Nat Clin Pract Endocrinol Metab 2: 269-281, 2006.

3. Sugimura K, Windebank AJ, Natarajan V, Lambert EH, Schmid HH and Dyck PJ: Interstitial hyperosmolarity may cause axis cylinder shrinkage in streptozotocin diabetic nerve. J Neuropathol Exp Neurol 39: 710-721, 1980.

4. Selagzi H, Buyukakilli B, Cimen B, Yilmaz N and Erdogan S: Protective and therapeutic effects of swimming exercise training on diabetic peripheral neuropathy of streptozotocin-induced diabetic rats. J Endocrinol Invest 31: 971-978, 2008.

5. Tesfaye S, Harris ND, Wilson RM and Ward JD: Exercise-induced conduction velocity increment: a marker of impaired peripheral nerve blood flow in diabetic neuropathy. Diabetologia 35: $155-159,1992$.

6. Calcutt NA, Jolivalt CG and Fernyhough P: Growth factors as therapeutics for diabetic neuropathy. Curr Drug Targets 9: 47-59, 2008 .

7. Chattopadhyay M, Mata M, Goss J, Wolfe D, Huang S, Glorioso JC and Fink DJ: Prolonged preservation of nerve function in diabetic neuropathy in mice by herpes simplex virusmediated gene transfer. Diabetologia 50: 1550-1558, 2007.

8. Mizisin AP, Calcutt NA, Tomlinson DR, Gallagher A and Fernyhough P: Neurotrophin-3 reverses nerve conduction velocity deficits in streptozotocin-diabetic rats. J Peripher Nerv Syst 4: 211-221, 1999.

9. Cuppini R, Sartini S, Agostini D, Guescini M, Ambrogini P. Betti M, Bertini L, Vallasciani M and Stocchi V: Bdnf expression in rat skeletal muscle after acute or repeated exercise. Arch Ital Biol 145: 99-110, 2007.

10. Ying Z, Roy RR, Edgerton VR and Gomez-Pinilla F: Voluntary exercise increases neurotrophin-3 and its receptor TrkC in the spinal cord. Brain Res 987: 93-99, 2003.

11. Qiu S, Wu C, Lin F, Chen L, Huang Z and Jiang Z: Exercise training improved insulin sensitivity and ovarian morphology in rats with polycystic ovary syndrome. Horm Metab Res 41: $880-885,2009$

12. de Oliveira CA, Luciano E and de Mello MA: The role of exercise on long-term effects of alloxan administered in neonatal rats. Exp Physiol 90: 79-86, 2005.

13. Zotova EG, Christ GJ, Zhao W, Tar M, Kuppam SD and Arezzo JC: Effects of fidarestat, an aldose reductase inhibitor, on nerve conduction velocity and bladder function in streptozotocin-treated female rats. J Diabetes Complications 21: 187-195, 2007.

14. Jiang Z, Kohzuki M,Harada T and Sato T: Glutathione suppresses increase of serum creatine kinase in experimental hypoglycemia. Diabetes Res Clin Pract 77: 357-362, 2007.

15. Said G: Diabetic neuropathy - a review. Nat Clin Pract Neurol 3: 331-340, 2007.

16. Azad N, Emanuele NV, Abraira C, Henderson WG, Colwell J, Levin SR, Nuttall FQ, Comstock JP, Sawin CT, Silbert C and Rubino FA: The effects of intensive glycemic control on neuropathy in the VA cooperative study on type II diabetes mellitus (VA CSDM). J Diabetes Complications 13: 307-313, 1999.

17. Balducci S, Leonetti F, Di Mario U and Fallucca F: Is a long-term aerobic plus resistance training program feasible for and effective on metabolic profiles in type 2 diabetic patients? Diabetes Care 27: 841-842, 2004. 
18. Castaneda C, Layne JE, Munoz-Orians L, Gordon PL, Walsmith J, Foldvari M, Roubenoff R, Tucker KL and Nelson ME: A randomized controlled trial of resistance exercise training to improve glycemic control in older adults with type 2 diabetes. Diabetes Care 25: 2335-2341, 2002.

19. Hoybergs YM, Biermans RL and Meert TF: The impact of bodyweight and body condition on behavioral testing for painful diabetic neuropathy in the streptozotocin rat model. Neurosci Lett 436: 13-18, 2008.

20. Tomlinson DR, Holmes PR and Mayer JH : Reversal, by treatment with an aldose reductase inhibitor, of impaired axonal transport and motor nerve conduction velocity in experimental diabetes mellitus. Neurosci Lett 31: 189-193, 1982.

21. Balducci S, Iacobellis G, Parisi L, Di Biase N, Calandriello E, Leonetti F and Fallucca F: Exercise training can modify the natural history of diabetic peripheral neuropathy. J Diabetes Complications 20: 216-223, 2006.

22. Buschbacher RM: Body mass index effect on common nerve conduction study measurements. Muscle Nerve 21: 1398-1404, 1998.

23. Landau ME, Barner KC and Campbell WW: Effect of body mass index on ulnar nerve conduction velocity, ulnar neuropathy at the elbow, and carpal tunnel syndrome. Muscle Nerve 32: 360-363, 2005.

24. Mandai K, Guo T, St Hillaire C, Meabon JS, Kanning KC, Bothwell M and Ginty DD: LIG family receptor tyrosine kinaseassociated proteins modulate growth factor signals during neural development. Neuron 63: 614-627, 2009.
25. Farinas I, Jones KR, Backus C, Wang XY and Reichardt LF: Severe sensory and sympathetic deficits in mice lacking neurotrophin-3. Nature 369: 658-661, 1994.

26. Lechner SG, Frenzel H, Wang R and Lewin GR: Developmental waves of mechanosensitivity acquisition in sensory neuron subtypes during embryonic development. EMBO J 28: 1479-1491, 2009.

27. Grill R, Murai K, Blesch A, Gage FH and Tuszynski MH: Cellular delivery of neurotrophin-3 promotes corticospinal axonal growth and partial functional recovery after spinal cord injury. J Neurosci 17: 5560-5572, 1997.

28. Nakahara Y, Gage FH and Tuszynski MH: Grafts of fibroblasts genetically modified to secrete NGF, BDNF, NT-3, or basic FGF elicit differential responses in the adult spinal cord. Cell Transplant 5: 191-204, 1996.

29. Schnell L, Schneider R, Kolbeck R, Barde YA and Schwab ME: Neurotrophin-3 enhances sprouting of corticospinal tract during development and after adult spinal cord lesion. Nature 367: 170-173, 1994.

30. Shibayama M, Hattori S, Himes BT, Murray M and Tessler A: Neurotrophin-3 prevents death of axotomized Clarke's nucleus neurons in adult rat. J Comp Neurol 390: 102-111, 1998.

31. Fernyhough P, Diemel LT and Tomlinson DR: Target tissue production and axonal transport of neurotrophin-3 are reduced in streptozotocin-diabetic rats. Diabetologia 41: 300-306, 1998. 